\title{
Estrategias de vigilancia policial en el Festival Internacional de Cine de Mar del Plata entre 1959 y 1960
}

Police surveillance strategies at the Mar del Plata Film Festival between 1959 and 1960

\section{Carlos García-Rivas}

300286475dalumnos.uc3m.es

Universidad Carlos III, Madrid, España

https://orcid.org/0000-0001-5920-7469

\section{Resumen}

La Guerra Fría desencadenó numerosos cambios radicales, tanto socioeconómicos como políticos, afectando a comunidades y a individuos en todo el mundo. En ese marco, las convulsiones macropolíticas de esas décadas se manifestaron también de forma concreta en los actores de la cultura cinematográfica de la Argentina. La vigilancia policial desplegada contra algunos personajes vinculados directa 0 indirectamente con el comunismo fue una de ellas. A través del análisis exhaustivo de los archivos policiales de la Dirección de Inteligencia de la Policía de la Provincia de Buenos Aires (DIPPBA), este artículo reconstruye las políticas de vigilancia desplegadas en el marco del Festival Internacional de Cine de Mar del Plata de 1959 y 1960. La DIPPBA individualizó y monitoreó a los invitados sospechosos de mantener relación con el comunismo. Las estrategias policiales se articularon gracias a los espacios que brindó el Festival de Cine de Mar del Plata.

Palabras clave: Argentina, vigilancia policial, archivo policial, festivales de cine, Mar del Plata.

\begin{abstract}
The Cold War unleashed several radical changes, both socio-economic and political, affecting communities and individuals all over the world. The macro political upheavals triggered by this global phenomenon affected workers of the cultural and film industry in a very concrete, daily-based way, in Argentina. The surveillance deployed by the police against some individuals directly or indirectly linked to communism was one of them. Based upon a close reading of the archives of the Board of the Police Intelligence of the Province of Buenos Aires (DIPPBA), this article depicts the surveillance tactics deployed by the police during the Mar del Plata International Film Festival of 1959 and 1960. The DIPPBA identified and monitored individuals suspected of having a relationship with communism. The police strategies were articulated under the organization provided by the Mar del Plata Film Festival.
\end{abstract}

Keywords: Argentina, police surveillance, police records, film festival, Mar del Plata. 


\section{Introducción}

A cinco días del inicio del Festival Internacional de Cine de Mar del Plata (FIMP) de 1959, la Oficina de Información Antidemocrática, a cargo del coronel Francisco Aintraz Galindes, envía el memorándum con fecha 6 de marzo de 1959 al Jefe de la Dirección Central de Inteligencia de Buenos Aires (DIPPBA), Antonio Piñeyro. En ella se advierte que, debido a la realización del festival de cine en la ciudad de Mar del Plata, llegarán a la Argentina delegaciones artísticas provenientes de países que se encontraban dentro de las influencias políticas e ideológicas comunistas ${ }^{1}$. En tal sentido, en dicho memorándum, se solicita "disponer de la vigilancia necesaria" para supervisar que los visitantes "se concreten pura y exclusivamente a la finalidad que se les concedió la visa solicitada" (Dirección de Inteligencia de la Policía de la Provincia de Buenos Aires [DIPPBA], 1959).

El inicio de estas vigilancias coincide con el segundo año del presidente Arturo Frondizi al mando del gobierno argentino. En el plano político argentino, 1959 fue un año de incremento del descontento popular. Las políticas de austeridad adoptadas por el gobierno de Frondizi y la intención de privatizar las empresas que anteriormente habían sido nacionalizadas por el gobierno de Juan Domingo Perón (1946-1955), generaron el descontento de comunistas y peronistas. Dicho malestar se manifestó, por ejemplo, en la denominada Batalla del Petróleo que se oponía a la firma de contratos de explotación petrolera con empresas multinacionales en detrimento de las industrias estatales, las huelgas del gremio de los banqueros en 1959 y huelgas en general que se oponían al Plan de Estabilización y Desarrollo, también conocido como "Plan de austeridad y sacrificio", ideado bajo los lineamientos del Fondo Monetario Internacional (FMI). Estos fueron algunos de los motivos de la creciente insurrección de grupos comunistas y peronistas que acusaban al gobierno de alinearse a las políticas americanas (Ortega, 2010; Barreneche, 2011). Ante esta creciente insurrección se aplicó el Plan Conmoción Interna del Estado (CONINTES), que consistía en una serie de disposiciones dirigidas desde el poder ejecutivo que habilitaron utilizar las fuerzas militares para reprimir las protestas estudiantiles, comunistas y peronistas. Estas acciones militares estaban fuera de las leyes que la constitución amparaba.
Latinoamérica no estuvo ajena a la influencia de las potencias que emergieron como ganadoras de la Segunda Guerra Mundial en su afán por copar todas las dimensiones posibles de la vida de las personas. Las naciones hegemónicas disputaron dicha influencia, intentando disminuir el poder de sus adversarios al mismo tiempo que trataban de fortalecer el propio. Al inicio, Latinoamérica tuvo un rol secundario en la Guerra Fría. Sin embargo, "la región empezó a convertirse en un escenario muy particular durante los años cincuenta, con la caída del gobierno guatemalteco en 1954 y con el triunfo de la revolución cubana, en las postrimerías de esa misma década" (Agüero, 2016, p.152).

Este artículo se enmarca en enfoques desarrollados por la denominada "nueva historia de la Guerra Fría". Como bien demuestran investigaciones recientes, esta perspectiva teórica se refiere a paradigmas e innovaciones historiográficas que proponen conceptualizaciones alternativas a la de una guerra bipolar. En efecto, la nueva historia de la Guerra Fría destaca el papel de los países periféricos o del tercer mundo como actores de primer orden en los procesos ocasionados por la Guerra $\mathrm{Frí}^{2}$. En esta perspectiva se ubican trabajos como A Century of Revolution (Grandin, Joseph, Rosenberg, Katz \& Olcott, 2010) cuya tesis sostiene que la Guerra Fría no fue el principal causante del incremento revolucionario que vivió América Latina, pero sí logró intensificar los procesos de décadas precedentes.

El inicio del FIMP de 1959 también coincide con la hipótesis de censura implícita que postula Ramírez-Llorens (2016), quien estudia la censura en Argentina entre de 1955 y 1973. Esta hipótesis surge del análisis de cómo se delinearon las políticas de promoción y prohibición en aquellos años. Según el autor, "se basó en la concepción que los distintos actores en pugna tenían en torno a los usos sociales del cine y (que) expresó las luchas por la legitimación de ese uso social frente al resto de los actores" (p. 34). Actores principales como el Estado, los católicos y los empresarios, quienes implementaron "las políticas de promoción y prohibición formales o informales" a las cuales se fueron adhiriendo o rechazando el resto de actores, en algunos casos, "logrando un consenso en torno al uso social del cine cómo sano esparcimiento". En este sentido, Ramírez-Llorens sostiene que los años de 1955 a 1966 constituyeron una etapa en la que se observa 
un proceso de conveniencia de intereses entre los grupos censores y empresarios cinematográficos. Durante el gobierno de Frondizi los grupos católicos tenían una gran influencia en las políticas educativas. Así, en esos primeros años del FIMP, las censuras eran de orden moral, educativo y económico o de promoción.

En este ambiente revuelto y de censura implícita en la industria cinematográfica, se realizaría la segunda edición del FIMP33 , organizada exclusivamente por la Asociación de Cronistas de la Argentina que estaba conformada por un grupo de cinéfilos y teóricos con motivaciones artísticas. Como recuerda Héctor Grossi, quien fuera jefe de prensa del Festival Internacional de Cine de Mar del Plata entre 1960 y 1964 :

... en 1959, el Festival Internacional de Cine de Mar del Plata, no tuvo una motivación ajena al cine mismo. Al cine como expresión artística y cultural... No fue un festival semejante al precedente argentino en 1954 que organizó el gobierno del general Juan Domingo Perón, concebido con astucia política y de propaganda agónica (Grossi, 1995, p. 241).

Las investigaciones que abordan el FIMP durante la década del sesenta mayoritariamente coinciden con el recuerdo de Grossi. En efecto, diversos autores sostienen que durante esos años primó la pasión por el cine en la organización y realización del festival (Manetti \& Valdez, 2005; Toribio, 2007). Dichos propósitos iniciales del FIMP son destacados, también, por los órganos oficiales de difusión del certamen, como la Gaceta del Festival número 5 de la versión de 1968 que hizo una retrospectiva del primer año del festival bajo la conducción de los cronistas, según cita Toribio (2007):

El festival del Mar del Plata, creado en 1959, tuvo desde su nacimiento propósitos bien definidos. Sin olvidar la necesaria presencia de figuras populares útil más que nada para mantener un cierto clima de excitación, el acento estuvo puesto siempre en la faz cultural y en la trascendencia al plano industrial del evento. Grandes directores, importantes teóricos y críticos influyentes enriquecieron con su presencia las distintas ediciones de la muestra. Gracias a su visita, la Argentina y su cinematografía quedaron ubicadas en el lugar que merecían dentro del periodismo fílmico internacional (p.36)
Al mismo tiempo que un espacio enfocado a la cultura y la industria cinematográfica local e internacional, el FIMP se convirtió en uno de los focos y espacios de vigilancia del gobierno argentino. ¿Por qué se ordenó esta vigilancia en el festival de 1959? Dicha vigilancia, ¿fue gatillada por el ambiente social convulso en el país?, son las preguntas que guían este trabajo.

\section{Los archivos policiales de la DIPPBA}

El Fondo de la Dirección de Inteligencia de la Policía de la Provincia de Buenos Aires, conocido por su abreviatura DIPPBA, fue cedido a la Comisión Provincial por la Memoria (CPM) en el año 2001 después que el Congreso aprobara la ley 12.642. Dicha regulación autoriza y otorga a la CPM la responsabilidad de custodiar y poner a disposición de la justicia estos archivos donde consta un pormenorizado registro de espionaje político y de persecución política en la provincia de Buenos Aires. Estos archivos son públicos desde el año 2003. Debido a su relevancia para recuperar y resguardar la memoria histórica de una época, la UNESCO declaró estos archivos como patrimonio de la humanidad en el año 2008.

El contenido sensible de estos archivos implica evaluar las políticas y estrategias más adecuadas para ponerlos a disposición del público y así lo ha entendido la CPM. En ese sentido, el trabajo realizado por Jaschek, Lanteri, Sahade y Soler (2018), integrantes de la CPM, han desarrollado una metodología que se adapte de la mejor manera a este material. Ellas proponen "tres estrategias posibles para pensar las políticas de acceso" a los archivos de la DIPPBA: en primer lugar, un "protocolo que dé pautas claras a los diferentes usuarios; en segundo lugar, es importante encontrar las herramientas archivísticas que describan y faciliten la comprensión del fondo; y una tercera estrategia que ayude a dar a conocer su existencia" (p.11). El estudio de estos archivos, de alguna manera, nos obliga a considerar el contexto histórico en el que se elaboraron, la historia de la institución y la intención "de 'memoria oficial' que tuvo en el momento de su producción". Tres puntos que ayudan a entender de la mejor manera su contenido (Jaschek et al., 2018, p. 11). 
Las publicaciones de los dossiers producidos para la revista Puentes a cargo de los integrantes de la Comisión Provincial por la Memoria se han agrupado en series documentales $y$, hasta la fecha de edición del presente artículo, en 15 publicaciones. Dichas series documentales están basadas en hechos, situaciones, acontecimientos históricos y temáticas como, por ejemplo, un partido de fútbol, la censura de ciertas músicas populares o diferentes acontecimientos referidos a la vigilancia dirigida hacia los jóvenes, entre muchos otros factores ${ }^{4}$.

Desde su creación en 1956, la DIPBA debía cumplir con ciertos requerimientos para su correcto funcionamiento. En ese marco, uno de sus objetivos era prevenir y reprimir la protesta social. La implementación de la Dirección, así como de sus funciones, estuvo a cargo de los militares, bajo la conducción del General Carlos Tomaso Montore, quien, con "una mentalidad maccartista" que "se apoderó de los organismos militares", exigió "reemplazar a las autoridades civiles en la represión del terrorismo y de las actividades subversivas" (Funes \& Jaschek, 2005, p.3).

En el Legajo No 25 Central de Inteligencia. Departamento " $C$ ", se establece que la recolección de información y el suministro oportuno a los organismos pertinentes son para "conjurar o contrarrestar una posible alteración pública en los órdenes: sindical, cultural, económico, político etc." Este plan de censura ideológico no podía ser público en 1957, ya que no estaban reprimidas las actividades comunistas. Bajo esa lógica, los organismos de inteligencia debían aprovechar la libertad de la cual gozaban adherentes o militantes comunistas para que se expresaran públicamente y, así, registrarlos, catalogarlos e incluirlos en la nómina de simpatizantes de la ideología marxista. La documentación consultada destaca que las tendencias comunistas y afines tenían intensa aceptación en esferas intelectuales, por lo que se recomendaba implementar una severa, a la vez que discreta, vigilancia en colegios, universidades e instituciones similares.

La percepción que tenían las fuerzas de seguridad del gobierno argentino sobre la relación entre comunistas y gremios, y que se refleja en los estatutos para el buen funcionamiento de la DIPPBA en el Legajo No 25 Central de Inteligencia. Departamento " $C$ ". Informaciones que se requieren para el normal desenvolvimiento (Funes \& Jaschek, 2005), era, pri- mero, que los comunistas responden a los intereses del partido antes que a los del gremio y, segundo, que éstos "tratan de generalizar todos los conflictos, para capitalizar los disturbios a su favor". Por lo tanto, las recomendaciones para el buen funcionamiento de la Dirección ordenan "ejercer control más estricto sobre las actividades comunistas... en los medios intelectuales y artísticos".

\section{Los espacios vigilados en el FIMP}

Los festivales de cine han sido conceptualizados como sites of passage, noción desarrollada por de Valck (2007). En efecto, estos sites of passage se refieren a que constituyen lugares de encuentro en un espacio y tiempo determinados, donde personas, prestigio y poder se encuentran. Espacios que, por lo demás, son inherentemente transnacionales debido a que forman parte de una red internacional de festivales, como refiere lordanova (2016).

Además, en estos espacios se observa una gran variedad de rituales como la alfombra roja, las ceremonias de inauguración y de premiación y, en general, un conjunto de actos simbólicos que hacen que las películas, los cineastas y realizadores en general se posicionen en el mundo cinematográfico. Esta característica de actuación ritualista crea en los festivales la idea de una suspensión en el tiempo, donde se crean reglas independientes a cada festival de cine y se suspenden las reglas del mercado cinematográfico exterior ${ }^{5}$.

El FIMP, en aquellos primeros años, fue delineando sus singulares características, facilitando las condiciones apropiadas que permitieron el encuentro e intercambio tanto comercial como cultural e ideológico. El certamen se constituyó como un espacio en el cual se dieron cita intelectuales de todas las categorías. Desde 1959, el FIMP se convirtió en un escenario de encuentro en el cual se hicieron visibles casos de censura. Por ejemplo, las primeras reuniones entre directores, actores y el Dr. Emilio Zolezzi como director del Instituto Nacional de Cine (INC), fueron instancias en las cuales se aclaraban las preocupaciones y dudas sobre la función que ejercía dicha institución. Al año siguiente, se realizaría, formalmente y por primera vez, el encuentro internacional entre reali- 
zadores, teóricos y personalidades del mundo del cine. Como recuerda Alsina Thevenet en sus artículos de la época y que recogen Buela, Gandolfo \& Peña (2012), dichas reuniones eran "el tipo de cosas que solo se puede hacer en un festival... porque en pocas ocasiones similares se podría juntar gente de tan alto nivel" (p. 649). Thevenet se refería a personalidades como los teóricos George Sadoul y George N. Fenin, la productora Kashiko Kawakita, los guionistas Derek Prouse y Beatriz Guido, el académico Narciso Pousa, el escenógrafo Saulo Benavente, el periodista Carlos Burone y los directores de cine Walter Hugo Khouri, Fernando Ayala y Torre Nilson, entre otros actores clave del campo.

La ciudad de Mar del Plata contaba con la infraestructura óptima para ser la sede de un evento internacional. Ya desde el festival de cine de 1954 organizado por el presidente argentino Juan Domingo Perón, Mar del Plata se convertiría en un espacio privilegiado para este tipo de eventos. Las instalaciones facilitadas para el FIMP eran diversas y fueron destinadas dependiendo de los eventos que alojasen. La sede principal era el Hotel Provincial con sus distintos salones preparados exclusivamente para los eventos oficiales como las fiestas de inauguración y de clausura. El cine Ocean Rex y Ópera eran los principales cines en los que se programaban las exhibiciones de la programación oficial.

Otra instalación relevante para el festival era la sede local del Automóvil Club Argentino. Allí se realizaban las conferencias de prensa de las delegaciones. Este lugar era uno de los más concurridos por periodistas y público en general que intentaba obtener un autógrafo. Entre las instalaciones disponibles se contaban, también, los auditorios y los salones de los teatros, entre los que se contaban el teatro Colón y el teatro Auditórium del Gran Casino, que alojaban los actos culturales como retrospectivas, exposiciones y encuentros. En las calles de la ciudad de Mar del Plata se celebraban, también, eventos al aire libre como el cine itinerante, las exhibiciones de películas, de autos antiguos y de fotografías, entre otras expresiones artísticas y culturales simultáneas y/o al alero del FIMP.

Todos estos lugares habilitados y dispuestos para los organizadores fueron diseñados para fomentar la mayor interacción entre sus invitados en los once días que duraba el certamen. Por lo tanto, los lugares debían estar lo más cerca posible los unos de los otros para, así, evitar que invitados, periodistas y público en general se perdieran algún acto programado. La logística debía garantizar que el festival fuera el lugar de encuentro y socialización entre las delegaciones de los distintos países congregados en Mar del Plata.

Alsina Thevenet, días antes del inicio del festival, veía con esperanzas la presencia del nuevo cine de Polonia y Hungría. En un artículo publicado el 2 de marzo de 1959, Thevenet destacaba "lo positivo que será para estos países presentarse al festival ya que les daba la oportunidad de expandirse por el continente sudamericano con mayor facilidad" (Buela et al., 2010, p. 726). La transnacionalidad en tanto característica propia de los festivales, como el de Mar del Plata, permitió que los espías y/o agentes encargados de las vigilancias tuvieran en un mismo espacio y tiempo determinados, reunidas e interactuando, a las delegaciones provenientes de los países de la llamada cortina de hierro (lordanova, 2016).

En los primeros memorándums se especifica la vigilancia a los miembros de las delegaciones de los países provenientes de países con regímenes comunistas y afines. Los primeros informes elaborados por la policía local de Mar del Plata incluyen una lista detallada de las delegaciones de Hungría, Checoslovaquia (actual República Checa), Polonia, Unión Soviética y Bulgaria. De Hungría, el reporte policial especifica que la comitiva se aloja en el hotel Provincial, los números de sus respectivas habitaciones y sus integrantes; sobre los checos, informa que están alojados en el hotel Nagaró; sobre la delegación polaca, el reporte policial detalla que se alojan en el hotel Hermitage, así como también indica sus respectivas habitaciones e integrantes. De la Unión Soviética y Bulgaria se informa que aún no han arribado a la ciudad de Mar del Plata. Se observa que los espías informan de países de gobiernos comunistas que aún no habían llegado y, en general, aclarando al final de los informes sobre la inactividad de algunos de ellos. Por el contrario, cuando hay movimientos se registra como “novedad".

Los miembros de la policía secreta no implementan sus vigilancias solo en las instalaciones de los hoteles en que se alojan las delegaciones, sino que, también, en los lugares donde decidieron 
hospedarse algunos de los integrantes de las delegaciones vigiladas, fuera de los hoteles oficiales. Por ejemplo, periodistas y delegados culturales decidieron alquilar un chalet ubicado a 10 kilómetros (20 minutos en automóvil) desde el lugar del evento. Los documentos de la Dirección registran la orden de procurar, "por todos los medios, su individualización y localización, a fin de su vigilancia y su seguimiento" (DIPPBA, 1959).

\section{La individualización y localización de los vigilados}

Los archivos también indican, por ejemplo, que los integrantes de la delegación húngara rechazaron los servicios de peluquería que la organización del festival brinda a todos los participantes y que estos están utilizando los servicios del artesano establecido con el negocio "Peinados Bernardo". Se menciona y destaca el origen soviético del dueño de dicho establecimiento $y$, por consiguiente, se ordena "se realicen las averiguaciones entorno de su identidad, ideología y forma de conducirse, a fin de informar al respecto" (DIPPBA,1959).

Identificar la identidad, la ideología y la forma de conducirse de los participantes del festival y de todos aquellos que tengan acercamientos con ellos y, en este caso, más aún debido a su origen soviético, proporciona al menos tres campos que abarcan un gran espectro de un individuo. Por lo tanto, si preguntamos: ¿Para qué se vigilaba?, las respuestas pueden ser: Para conocer la identidad de las personas relacionadas con ideas comunistas que se encontraban en el territorio argentino, saber sus nombres, procedencias y otros datos personales; conocer su ideología, es decir, averiguar las ideas que los identifican o dan pertenencia dentro de una colectividad, ya sea religiosa, cultural o política, y, finalmente, para identificar sus conductas, es decir, cómo se comportan o reaccionan frente a ciertas circunstancias ${ }^{6}$.

Los informes policiales registran, además, percepciones que exceden los datos o características de los vigilados e ilustran la subjetividad de los informantes. Dichos informantes, a partir de características objetivas de quienes estaban bajo vigilancia, especulaban sobre diversos imaginarios sobre los individuos a quienes espiaban. En los folios 14 al 16 de los archivos DIPPBA, se incluye una descripción física respecto de la cual la subjetividad de los espías jugaba un papel importante. En el caso del vocal del Jurado Cinematográfico Pousa Narciso, podemos observar los datos objetivos y la información subjetiva que los espías realizaban:

\begin{abstract}
Con respecto a las averiguaciones practicadas para con el señor Pousa Narciso que se aloja en el departamento 571 del hotel Provincial desde el día 11 del corriente, el mismo es profesor de castellano y representante de Lenguas vivas siendo en la actualidad vocal de la comisión del Jurado Cinematográfico. Sus antecedentes personales son el siguiente: Casado; con dos hijos y domiciliado en la calle 56 aproximadamente entre 12 y 13 de la ciudad de la Plata. La posición económica del Señor se estima que es por demás desahogada, tratándose de un individuo de un porte elegante, pero de ciertas inclinaciones que lo sindican como pederasta pasivo, dato éste que se le puede dar crédito dado el origen de las informaciones obtenidas.
\end{abstract}

En este caso, puede conocerse la identidad y suponer ciertas conductas privadas a partir de algunas características físicas o ciertas conductas con respecto a quienes rodean a un vigilado. Sin embargo, identificar o suponer la ideología de los espiados no es fácil, requiere el empleo de técnicas de espionaje que superan las especulaciones y que las vigilancias hasta el momento no logran. Se ve entonces la necesidad de infiltrar a los espías en las reuniones y en los espacios que ocupan las delegaciones invitadas.

Esta infiltración en diversos espacios e instancias del festival recoge conversaciones entre los delegados y sobre el trato que se daban mutuamente. Este tipo de reportes dan a entender lo cerca que debían permanecer los espías de sus objetivos. Los puntos de encuentro más concurridos por los espías eran los hoteles Hermitage y Provincial, recintos donde se alojaban principalmente las delegaciones de los países de la cortina de hierro y donde se realizaban las recepciones y reuniones informales entre los participantes del festival. Por ejemplo, las actrices argentinas Nelly Kaplan y Lidia Elsa Vatragno, según los informes de inteligencia, se les observaba conversando a menudo con las delegaciones de Hungría y Polonia. Pero, según narran los agentes, no era posible obtener información debido 
a que el idioma con el que se comunicaban era desconocido para los espías.

Ante este impasse, los espías revisaron los equipajes de las actrices mencionadas. Es decir que las vigilancias no solo se realizaban en los espacios públicos, sino que también, como explicitan los reportes revisados, los agentes tenían acceso a los equipajes. No se especifica si entraron a las habitaciones, pero de los informes se desprende que los espías se infiltraban y navegaban tanto en los espacios públicos en los que transcurría el festival, así como también en los ámbitos más privados de sus objetivos, en este ejemplo, el equipaje de las actrices. Informan que realizaron "una prolija inspección en los equipajes de todas las personas mencionadas pero sin obtener dato de interés" (DIPPBA, 1959). Este nivel de infiltración, que menciona una prolija inspección, supone que los agentes destinados al seguimiento y vigilancia de los asistentes al FIMP contaban con facilidades para acceder e intervenir objetos y espacios personales, así como tiempo y comodidad suficientes para cumplir las directrices del organismo.

Tabla 1: Datos objetivos y subjetivos en los memorándum

\begin{tabular}{l|l|l} 
Nombre & Pousa Narciso & Información Subjetiva \\
\hline Situación en el festival & Información Objetiva & La posición económica se estima \\
\hline $\begin{array}{l}\text { Departamento número 571 } \\
\text { del hotel Provincial. } \\
\text { Día de llegada el día } 11 \text { de Marzo de 1959. }\end{array}$ & $\begin{array}{l}\text {-Profesor de castellano y vocal } \\
\text { del Jurado Cinematográfico. } \\
\text { individuo de un porte elegante, } \\
\text { pero con cierta inclinación que lo } \\
\text { sindica como pederasta pasivo. }\end{array}$ \\
\hline
\end{tabular}

Fuente: elaborada por el autor basado en la información de los folios 14 y 15 del Archivo DIPPBA, "Festival Internacional Mar del Plata 1959".

\subsection{Las estrategias de vigilancia}

Para el festival de 1960 los preparativos de vigilancia comenzaron el 3 de diciembre de 1959. En esa fecha, el comisario Horacio Pedro Ochando solicita antecedentes de toda índole que pudieran registrarse en la Policía Federal sobre los invitados confirmados al FIMP. La solicitud está dirigida a la central de la DIPPBA. En esta oportunidad, el objetivo era estar prevenido de algún visitante que requiera vigilancia especial.

Ochando justifica su pedido explicando que, en la versión previa del certamen, se había observado "una evidente preponderancia de elementos comunistas" (DIPPBA,1959) y no se tomaron los resguardos correspondientes para implementar a tiempo mecanismos de vigilancia. En efecto, el año anterior se registraron ciertos hechos sobre los cuales el espionaje no fue fructífero pues no se planificaron ciertas medidas o acciones para abordarlos. Posteriormente, cuando la Secretaría de Información del Estado, a través del departamento de Informaciones Antidemocráticas, solicitó información sobre algunos sucesos en los que se observaban componentes comunistas, ya los hechos no se podían rastrear.

Un ejemplo de aquello se encuentra en los hechos ocurridos en las inmediaciones del hotel Hermitage y del cine Ocean Rex. Allí, algunas actividades llamaron la atención de los espías: Dos jóvenes de sexo masculino de aparente nacionalidad argentina, de aproximadamente 20 y 25 años, que anteriormente habían sido observados en el hotel Hermitage, lugar donde se habían reunido con la delegación de Polonia y que se identificaron como amigos de dicha delegación sin dar sus nombres, se les volvía a ver en las inmediaciones del cine Ocean Rex reunidos con la misma delegación, don- 
de permanecieron hasta las 3:15 horas de la madrugada, "evidenciando entenderse sin dificultades con los integrantes de la delegación, retirándose finalmente en un automóvil con patente de la capital federal 520.603" (DIPPBA, 1959). De todos los memorandos enviados por los espías, este que se envió con fecha 19 de marzo de 1959, el mismo día de los hechos, fue el que llamó la atención de la Secretaría de Información del Estado. La Secretaría pidió más información sobre los hechos sucedidos, además de solicitar que se identificara a los jóvenes, pedido que la delegación DIPPBA de Mar del Plata no pudo satisfacer.

En consecuencia, la información solicitada con antelación debía prevenir a los espías en sus funciones de vigilancia y evitar que se repitieran sucesos como los descritos en el párrafo anterior de los cuales los espías no pudieran responder. El informe de la Policía Federal de la delegación de La Plata llegaría después de varios intentos por conseguir una respuesta a los datos solicitados. Se deja constancia de las reiteradas llamadas que se han realizado insistiendo por el informe con los antecedentes de los invitados confirmados al FIMP. No fue sino hasta el 25 de enero de 1960, casi dos meses después de la solicitud y alrededor de un mes y medio antes del inicio del festival, cuando se envían los resultados de la consulta.

\subsubsection{El caso de Saulo Benavente}

En la información producida por la Policía Federal, de las 14 personas mencionadas, solo una tenía antecedentes policiales. En la lista solo figuran dos personas con parientes con algún pequeño incidente policial. Sin embargo, de igual manera, se detalla información de las personas que la lista contenía. En el folio 52 del 25 de enero de 1960, perteneciente al legajo 12218 del archivo DIPPBA. Por ejemplo, se describe al escenógrafo Saulo Benavente, que después de describir la nacionalidad, documentos de identidad, domicilio, profesión y parientes cercanos, se detalla las actividades en las que había participado.

Se dice que el año 1953 se comprometió a participar como colaborador del Congreso Cultural Americano a celebrarse en Santiago de Chile entre los días 26 del mes de abril y el 4 de mayo de 1953, siendo este congreso "de carácter comunista".
Además se agrega que participó en el Congreso de la Juventud realizado en Bucarest en Junio de 1953 figurando, además, como vicepresidente de la revista Cultura China en la que se destaca la tarea realizada por el gobierno de la República Popular China, alabando al régimen comunista.

En 1954 se tiene registrado en un informe confidencial del 28 de septiembre que Frantissek Hrabalek, empleado de la delegación checoslovaca, mantuvo contacto con Saulo Benavente. El informe también hace referencia a un artículo aparecido en el diario La Prensa del 4 de abril de 1955, el que informa que la Asociación de Cultura China, bajo la dirección del escenógrafo Saulo Benavente, ha comenzado los trabajos de organización de su teatro.

En 1957, el 12 de abril es detenido acusado de actividades que atentan contra la seguridad del Estado, pero fue liberado siete días después. Con fecha 30 de agosto del mismo año, es mencionado en la revista Festival, como integrante del comité nacional preparatorio, constituido con el fin de enviar una delegación al VI Festival Mundial de la Juventud, a realizarse en Moscú. Con ese motivo Benavente habría realizado un viaje a China comunista.

En 1958 se informa que Benavente, según la publicación del diario La Hora, figura en el homenaje a María Rosa Lucia Oliver, con motivo de la entrega del premio internacional Lenin del 9 de junio de 1958. De acuerdo con la publicación del diario Correo de la Tarde del 3 enero de 1959, Benavente es el encargado de la escenografía de la pieza de Bertold Brecht, El Alma Buena de Se-Chuan, donde su nombre aparece junto a José Franyie, conocido miembro del Partido Socialista Popular de Cuba. En mayo del mismo año, figura en documentación incautada en la Liga Argentina por los Derechos del Hombre, donde aparece en una credencial como delegado en representación de Junta Capital ante el encuentro latinoamericano del 10 de julio de 1959, donde concurre a un cóctel servido en la Embajada Soviética en conmemoración de la Revolución Rusa (DIPPBA, 1960).

Con estos informes detallados de algunos participantes, el equipo de espías y/o empleados, como se les refiere en los memorándums, estaba preparado para recibir a los invitados del festival de 1960. A diferencia del primer año, ahora manejaban más 
información sobre a quienes debían prestar atención. De este modo, el 8 de marzo del mismo año, el empleado individualizado solo por el apellido Ruiz informaba de la llegada del primer tren en arribar a la ciudad de Mar del Plata y que transportaba las delegaciones de Alemania, Brasil y Estado Unidos.

Apenas un día después de la llegada de los invitados a la ciudad, la policía secreta contaba con la relación exacta de la identificación y ubicación de cada uno de los miembros de las delegaciones participantes del certamen. Se especifica el hotel de los invitados, los números de las habitaciones y los países de procedencia de cada uno. Junto a este informe se adjunta en el memorándum el programa de actos oficiales del FIMP, el calendario de las categorías de las películas participantes, las fechas de la realización de actos culturales, las proyecciones cinematográficas al aire libre, los actos patrocinados por la lotería de beneficencia nacional y casinos, los eventos organizados por la Comisión de Mar del Plata y las distintas y numerosas conferencias de prensa.

\section{Conclusión}

El análisis de los archivos de la Dirección de Inteligencia de la Policía de la Provincia de Buenos Aires (DIPPBA) permite identificar y reconstruir las prácticas de vigilancia policial e ideológica desplegadas durante el Festival Internacional de Cine de Mar del Plata en plena Guerra Fría (1959 y 1960). La vigilancia como consecuencia de un momento histórico y político que se vivió en Argentina, en particular, y en Latinoamérica, en general, que, en los últimos años y gracias a las desclasificaciones de archivos locales e internacionales, ven la luz y permiten (re)conocer nuevas perspectivas sobre Latinoamérica en el contexto de la Guerra Fría. Estas perspectivas novedosas bajo las cuales interpretar esos momentos, esos hechos, se inscriben en las propuestas como la de Joseph, Spenser y Rosenberg (2008) en In From the Cold: Latin America's New Encounter with the Cold War, libro fundacional que propone redirigir la atención desde una visión bipolar de la Guerra Fría hacia una que, por el contrario, se centre en conflictos locales y en las relaciones entre países latinoamericanos.
Como hemos visto, la paranoia comunista que se vivía en Argentina, como consecuencia de las políticas estadounidense por expandir su influencia en América Latina, ocasionó que las vigilancias estuvieran enfocadas no solo en las delegaciones de artistas provenientes de los países que conformaron la denominada cortina de hierro sino que, también, en individuos o personajes de la cultura local argentina, para lo cual se aplicaron estrategias de individualización y ubicación de los asistentes al FIMP con el objetivo de catalogarlos como comunistas. Además, se observa el uso de técnicas, como la infiltración y el seguimiento de "personas de interés", con el objetivo de mejorar la calidad de la información vertida en los memorándums, objetivo que pudo realizarse gracias a las singulares características del FIMP que reunía en un mismo espacio y tiempo a distintos sujetos de interés para la policía secreta local.

La potencialidad, así como los límites de los archivos de la DIPPBA, están siendo ampliamente estudiados, identificando nuevas perspectivas y campos en los que el sistema represivo y de control del gobierno argentino se desplegó. Este trabajo no es más que un pequeño aporte a la variedad de temas que proporciona el contenido de estos archivos. Se suma, así, a otras investigaciones basadas en archivos desclasificados, con el fin de contribuir a futuras investigaciones que busquen preservar la memoria histórica del cine argentino y latinoamericano y comprender, mejor, sus contextos de producción y circulación.

\section{Notas}

1. También llamada Telón de Acero y que fue pronunciada en el discurso que diera.

2. Para mayor información sobre las perspectivas para el estudio de la Guerra Fría en las que se basa este artículo, ver Stonor (2001), Grandin et al. (2010), Gaddis (2012) e Iber (2015).

3. Es considerada la segunda edición del festival de categoría internacional. Sin embargo, puede considerarse la tercera versión siguiendo a Fernando Martín Peña. El autor sostiene que la primera edición del festival de Cine de Mar del Plata es la de 1948, aunque no fuese competitivo ni tuviera carácter internacional (Peña, 2015, p. 43). 
4. Se puede encontrar más información sobre el avance de las investigaciones de los archivos de la DIPPBA en Funes (2004), Castronuovo \& Marengo (2015), la Comisión Provincial por la Memoria (2015) y Lanteri (2009).

5. Siguiendo a de Valck (2007), se crea un espacio en “estado liminal, donde los productos cinematográ- ficos se regodean de atención que reciben por sus logros estéticos, especificidad cultural o relevancia social" (p. 39).

6. Basado en los significados de los conceptos que da la RAE a identidad, ideología y conducta https://dle. rae.es/identidad.

\section{Referencias}

Agüero, J. (2016). América latina durante la guerra fría (1947-1989): Una introducción. InterSedes, 17(35), 151-195. https://doi.org/10.15517/isucr.v17i35.25566

Applebaum, A. (2014). El telón de acero: La destrucción de Europa del Este 1944-1956. Madrid: Penguin Random House.

Barreneche, 0. (2011). Paro de y represión a... policías reclamos salariales, protestas y huelga en la policía bonaerense (1955-1973). Desarrollo Económico, 51(202/203), 221-239. https://www.jstor.org/stable/23612382

Buela, A., Gandolfo, E. \& Peña, F. M. (Comps.) (2010). Homero Alsina Thevenet-Obras Incompletas Tomo II-A. Buenos Aires: Festival Internacional de Cine de Mar del Plata.

Buela, A., Gandolfo, E. \& Peña, F. M. (Comps.) (2012). Homero Alsina Thevenet-Obras Incompletas Tomo II-B. Buenos Aires: Festival Internacional de Cine de Mar del Plata.

Castronuovo, S. \& Marengo, M. (2015). El archivo policial como espacio de memoria: un sondeo por el ex archivo de la DIPPBA. Revista Electrónica de Fuentes y Archivos (REFA), (6), 106-125. https://refa.org.ar/file.php?tipo=Contenido\&id=142

CPM-FONDO DIPPBA. (1957). División central de documentación, Registro y Archivo, Legajo No 25 Central de Inteligencia. Departamento "C". Informaciones que se requieren para el normal desenvolvimiento.

CPM-FONDO DIPPBA. (1959). División central de documentación, Registro y Archivo, Mesa Referencias, Legajo No 12218.

CPM-FONDO DIPPBA. (1960). División central de documentación, Registro y Archivo, Mesa Referencias, Legajo No 12218.

CPM (2015). Historia institucional de la DIPPBA. La inteligencia policial a través de sus documentos. https://www.comisionporlamemoria.org/archivos/archivo/historia-institucionaldippba/historia-institucional-dippba.pdf

De Valck, M. (2007). Film Festivals: From European Geopolitics to Global Cinephilia. Amsterdam: Amsterdam University Press.

Funes, P. (2004). Medio siglo de represión. El Archivo de la Dirección de Inteligencia de la Policía de la Provincia de Buenos Aires. Revista Puentes, 11(4), 34-43.

Funes P. \& Jaschek, I. (2005). Dossier de documentos. De lo secreto a lo público. La creación de la DIPPBA. Revista Puente, 6(16), 65-72. http://www.comisionporlamemoria.org/ archivos/archivo/de-lo-secreto-a-lo-publico/dossier3.pdf 
Gaddis, J. L. (2012). Nueva historia de la Guerra Fría. México D.F: Fondo de Cultura Económica.

Grandin, G., Joseph, G. M., Rosenberg, E. S., Katz, F. \& Olcott, J. (2010). A century of revolution. North Carolina: Duke University Press Books.

Grossi, H. (1995). "Los cronistas y el Festival Internacional de Mar del Plata". En Ferreira, F. (Ed), Luz, cámara ... memoria (pp. 241-246). Buenos Aires: Corregidor.

Iber, P. (2015). Neither peace nor freedom: The cultural cold war in Latin America. Cambridge, Mass: Harvard University Press.

Iordanova, D. (2016). "The Film Festival and Film Culture's Transnational Essence". En M. de Valck, B. Kredell \& S. Loist (Eds.), Film festivals, history, theory, method, practice (pp. xi-xvii). New York: Routledge.

Jaschek, I., Lanteri, M., Sahade J. \& Soler, E. (2018). La Comisión Provincial de la Memoria y el fondo documental de la Dirección de Inteligencia de la Policía de la Provincia de Buenos Aires (DIPPBA). Tres estrategias posibles para pensar las políticas de acceso. Hilos Documentales, 1(1). http://sedici.unlp.edu.ar/handle/10915/71631

Joseph, G. M., Spenser, D., \& Rosenberg, E. S. (2008). In from the cold. North Carolina: Duke University Press Books.

Manetti, R. \& Valdez, M. (2005). "El festival de Mar del Plata (1959-1970)". En España, C. Cine argentino. Modernidad y vanguardias 1957-1983, volumen II (pp. 488-497). Buenos Aires: Fondo Nacional de las Artes.

Ortega, F. (2010). De la modernización a la racionalización. Políticas adoptadas por Frondizi ante la crisis ferroviaria (1958-1962). H-industrida: Revista de Historia de la Industria, los Servicios y las Empresas en América Latina, (7), 1. http://ojs.econ.uba.ar/index.php/H-ind/ article/view/422

Peña, F. (2015). "Aquello que amamos". En D. Kosak (Ed), La imagen recobrada: la memoria del cine argentino en el Festival de Mar del Plata (pp. 43-55). Buenos Aires: Instituto Nacional de Cine y Artes Audiovisuales.

Ramírez-Llorens, F. (2016). Noches de sano esparcimiento. Buenos Aires: Libraria.

Stonor, F. S. (2001). La CIA y la guerra fría cultural. Madrid: Debate.

Toribio, N. T. (2007). El festival de los cinéfilos transnacionales: Festival Cinematográfico Internacional de la República Argentina en Mar del Plata, 1959-1970. Secuencias. Revista de historia del cine, (25), 25-45. https://revistas.uam.es/secuencias/article/view/4080

\section{- Sobre el autor:}

Carlos García-Rivas es Doctor por la Universidad Carlos III de Madrid, Magíster en Investigación Aplicada a Medios de Comunicación y en Dirección de Empresas Audiovisuales. Graduado de la Pontificia Universidad Católica del Perú. Líneas de Investigación: historia del cine latinoamericano.

\section{- ¿Cómo citar?}

García-Rivas, C. (2020). Estrategias de vigilancia policial en el Festival Internacional de Cine de Mar del Plata entre 1959 y 1960. Comunicación y Medios, (42), 85-95. https://doi. org/10.5354/0719-1529.2020.57282 